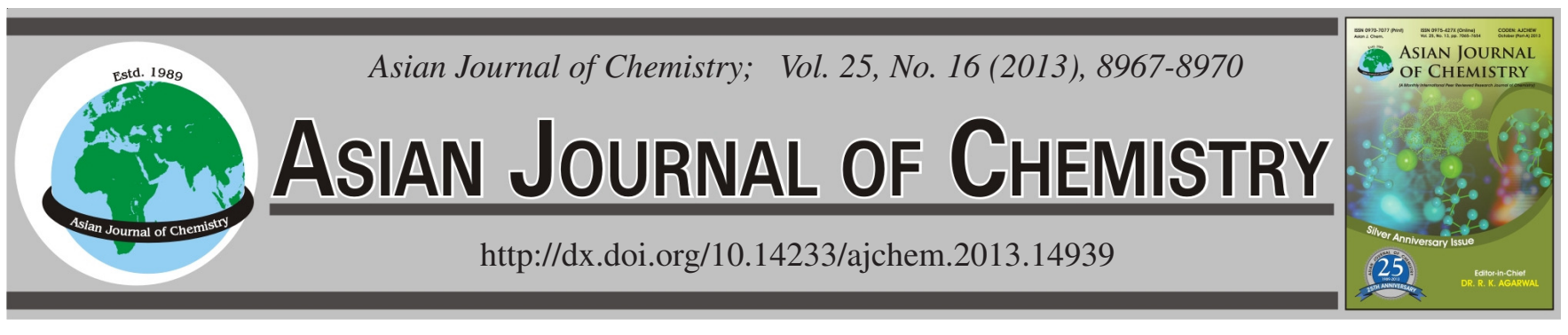

\title{
Simultaneous Determination of Tropane Alkaloids in Different Fractions of Herba belladonnae Collected in Various Seasons by HPLC Method
}

\author{
Wei Huang ${ }^{1}$, Jie Gao ${ }^{2}$, Weiming Cheng $^{3}$, Yingying Shangguan ${ }^{1}$, Xiaoyan Shou $^{1}$ and Wenting Zhang,
}

\begin{abstract}
${ }^{1}$ Zhejiang Medical College, No. 481, Binwen Road, Binjiang Dist. 310004, Hangzhou Zhejiang Province, P.R. China ${ }^{2}$ Zhejiang University of TCM, No. 548, Binwen Road, Binjiang Dist. 310053, Hangzhou Zhejiang Province, P.R. China

${ }^{3}$ Department of Traditional Chinese Medicine, Zhejiang Institute for Food and Drug Control, No. 86, Lane 1, Jichang Road, Jianggan Dist. 310004, Hangzhou Zhejiang Province, P.R. China
\end{abstract}

*Corresponding author: Tel: +86 13757170891; E-mail: leozhwt@163.com

\begin{abstract}
An HPLC method was developed for the simultaneous determination of hyoscyamine, scopolamine and anisodamine to investigate medical parts of Herba belladonnae in different harvest time. The solid phase was Shim-pack VP-ODS (4.6 $\mathrm{mm} \times 250 \mathrm{~mm}, 5 \mu \mathrm{m})$, with isocratic elution using $0.1 \%$ phosphoric acid solution (including $0.25 \%$ SDS)-acetonitrile (60:40) as the mobile phase. The flow rate was $1 \mathrm{~mL} / \mathrm{min}$ and the wavelength was $210 \mathrm{~nm}$. The results of methodology all fit the analytical requests and the recovery were $97.3-103.0 \%$. Quantitative analysis of the three compounds showed that the contents of hyoscyamine in leaves and flowers were the highest, followed by fruits and roots and the third was stem. The contents of scopolamine in flowers and fruits were the highest. The contents of anisodamine in fruits were the highest. The relative contents of hyoscyamine showed an increasing trend during the growth period, while scopolamine and anisodamine showed an opposite trend, which would reach a balance finally. The method was simple, quick and reliable and could provide a scientific and technical platform for setting up a quality control standard.
\end{abstract}

Key Words: Herba belladonnae, Hyoscyamine, Scopolamine, Anisodamine, HPLC.

\section{INTRODUCTION}

Herba belladonnae (HB) is the entire plant of Atropa belladonna, belonging to the family of Solanaceae ${ }^{1}$. Original from Europe, the main active components of Herba belladonnae are tropane alkaloids, possessing pharmacological actions of relieving smooth muscle spasm, inhabiting the secretion of glandular organs, etc. Clinically, it is mainly used for the treatment of hyperchlorhydria-induced duodenal ulcer and colicky pains on gastrointestinal tract, kidney and gall-bladder ${ }^{3,4}$. However, taking in excessive Herba belladonnae might result in serious toxic effects. Frequently, it has been reported poisoning incidents abroad due to eating the fruits of Herba belladonnae by mistake ${ }^{5-10}$. Herba belladonnae has been introduced into China since the 20th century, mainly used as the raw material for extracting belladonna extract and belladonna liquid extract. Because of the limitation of climatic condition in China, there are only certain major regions available for its plantation and cultivation. The researches of alkaloids in different medicinal parts changing along with different growing seasons are almost blank. Because of the lacking of scientific evidence on implantation and harvest, the usage and dosage of the herb are of indeterminacy. In addition, there is no report about the comparative analysis of alkaloids in different medicinal parts and different growing seasons yet. In this research, an HPLC method was developed primarily for the simultaneous determination of hyoscyamine, scopolamine and anisodamine and the changing rules of the above three tropane alkaloids in different medicinal parts and different growing seasons were summarized for the first time, which could provide reasonable evidence for its harvest and collection.

\section{EXPERIMENTAL}

Standards of anisodamine, scopolamine and hyoscyamine were supplied by Zhejiang Institute for Food and Drug Control (Hangzhou, Zhejiang Prov., China). Herba belladonnae was purchased from Hunan, China. Methanol and acetonitrile were of HPLC grade and other reagents used were analytical grade. Deionized water was prepared using a Millipore water purification system.

HPLC conditions: An Agilent 1200 series LC system was employed in this research, consisting of a G1379B Quaternary Pumps, a G1376B Degasser, a G1316A Diode-Array Detector and a G1376B Autosampler. 
The analysis of the alkaloids was carried out on a Shimpack VP-ODS $(250 \mathrm{~mm} \times 4.6 \mathrm{~mm}, 5 \mu \mathrm{m})$, protected by a Security GuardTM RP18 guard column ( $\mathrm{mm} \times 3.0 \mathrm{~mm}$ I.D., Phenomenex, USA).

The solvents used for HPLC separation of the three tropane alkaloids in samples were acetonitrile (A) and buffer solution (B, $0.004 \%$ phosphoric acid solution (including $0.25 \% \mathrm{SDS})$ ) at a flow rate of $1.0 \mathrm{~mL} / \mathrm{min}$. The mobile phase was isocratic elution with A-B (40:60, v/v) and the analysis was monitored at $210 \mathrm{~nm}$. The column temperature was $30^{\circ} \mathrm{C}$ and the sample injection volume was $10 \mu \mathrm{L}^{11}$.

Preparation of sample solutions: Medical parts of Herba belladonnae in different growing time were pulverized into powder, then passed through a $0.45 \mathrm{~mm}$ sieve, $c a$. $1.0 \mathrm{~g}$ sample was accurately weighted, then added into a $100 \mathrm{~mL}$ conical flask. $25.0 \mathrm{~mL} 50 \%$ methanol solution was added for ultrasonic batch at room temperature for $1 \mathrm{~h}$. The solution was ready for the chromatographic analysis after passing through a $0.45 \mu \mathrm{m}$ membrane filter.

Preparation of standard solutions: Three standard solutions, reference compounds anisodamine $(20.16 \mathrm{mg})$, scopolamine $(16.08 \mathrm{mg}$ ) and hyoscyamine $(24.40 \mathrm{mg}$ ) were dissolved with $50 \%$ methanol and diluted to five different concentrations.

\section{RESULTS AND DISCUSSION}

Regression equations: Linear regression analysis for each of the three tropane alkaloids was performed by the external standard method. Calibration curves were established based on six points for anisodamine with concentrations of 80.64 , 161.28, 322.56, 483.84645 .12 and $725.76 \mu \mathrm{g} / \mathrm{mL}$; six points for scopolamine with concentrations of $96.48,192.96,385.92$, $578.88,771.84$ and $868.32 \mu \mathrm{g} / \mathrm{mL}$; six points for hyoscyamine with concentrations of 195.2, 390.4, 780.8, 1366.4, 1561.6 and $1756.8 \mu \mathrm{g} / \mathrm{mL}$. The calculated results were given in Table-1. All the alkaloids showed good linearity in a relatively wide concentration range.

TABLE-1

LINEAR REGRESSION EQUATION AND LINEAR RANGES

\begin{tabular}{llcc}
\hline Alkaloids & Regression equation & $\begin{array}{c}\text { Correlation } \\
\text { coefficient }\left(\mathrm{R}^{2}\right)\end{array}$ & $\begin{array}{c}\text { Linear range } \\
(\mathrm{mg} / \mathrm{mL})\end{array}$ \\
\hline Anisodamine & $\mathrm{Y}=1080.2 \mathrm{X}-1742.5$ & 0.9997 & $80.64-725.76$ \\
Scopolamine & $\mathrm{Y}=1000.6 \mathrm{X}-3122.2$ & 1.0000 & $96.48-868.32$ \\
Hyoscyamine & $\mathrm{Y}=122.2 \mathrm{X}+53.6$ & 0.9995 & $195.2-1756.8$ \\
\hline \multicolumn{2}{l}{ Note: $\mathrm{X}$ denoted the concentrations and $\mathrm{Y}$ denoted the peak areas. }
\end{tabular}

Precision: The standard mixture solution of anisodamine hydrobromide, Scopolamine hydrobromide and hyoscyamine sulfate was injected into HPLC for six times continuously and the area of each peak was used for the calculation of precision. The results showed that relative stand deviation (RSD) of peak area of each standard was $0.1,0.2$ and $0.5 \%$, respectively.

Repeatability: Repeatability was carried out taken six samples solution with the same treatment procedure. The results showed that RSD of each peak area was all $0.9 \%$.

Stability: For stability test, the same sample solution was analyzed at the designated time points for $48 \mathrm{~h}$. The results showed that RSD of peak area were $0.2,0.5$ and $0.2 \%$, stable for the experiment.
Recovery test: The sample with determined targeted contents was spiked with certain amounts of the 3 standards. Then the spiked sample was processed in accordance with the established method for HPLC detection. The average recoveries for anisodamine hydrobromide, scopolamine hydrobromide and hyoscyamine sulfate determined were 97.3-103.0\% (Table-2). Determination of tropane alkaloids in different fractions of Herba belladonnae are given in Table-3.

Application of the HPLC method for quantitation studies: $10 \mu \mathrm{L}$ sample solution was injected into the instrument. The representative HPLC chromatograms were shown in Fig. 1, respectively. Peaks in the obtained chromatograms were identified by comparing the retention time and on-line UV spectra with those of the standards.



Fig. 1. Typical HPLC chromatograms of standard solution (A) and sample solution (B). 1. Anisodamine hydrobromide. 2. Scopolamine hydrobromide. 3. Hyoscyamine sulfate

Optimization of HPLC separation conditions: In order to get a separation with better resolution of targeted components in a shorter analytical time, we compared three different column temperature: 20,30 and $40^{\circ} \mathrm{C}$, then we found that the higher temperature, the shorter retention time with almost the same resolution, so we chose $30^{\circ} \mathrm{C}$ finally for protecting the lifespan of the column.

Besides, reflux extraction and ultrasonic extraction were investigated in the experiments. Considering the advantages of high efficiency and easy operation, we chose ultrasonic extraction as the way to prepare sample solution. In the meantime, we compared the efficiency of $50 \%$ methanol with ethyl acetate which was used for ultrasound, it showed that the former was better. We also tried different ultrasonic time i.e., 30, 60 and $90 \mathrm{~min}$. Finally, taking the determination of targeted components into consideration, ultrasonic extraction $1 \mathrm{~h}$ with $50 \%$ methanol was better.

Three chromatographic columns were compared in the experiment: Agilent Zorbax SB-C ${ }_{18}(250 \mathrm{~mm} \times 4.6 \mathrm{~mm}, 5 \mu \mathrm{m})$, Kromasil 100-5C $18(250 \mathrm{~mm} \times 4.6 \mathrm{~mm}, 5 \mu \mathrm{m})$ and Shim-pack VP-ODS $C_{18}(4.6 \mathrm{~mm} \times 250 \mathrm{~mm}, 5 \mu \mathrm{m})$, according to the effect of separation, Shim-pack VP-ODS $\mathrm{C}_{18}(4.6 \mathrm{~mm} \times 250 \mathrm{~mm}$, $5 \mu \mathrm{m})$ was used for further research of the methodology. 


\begin{tabular}{|c|c|c|c|c|c|c|}
\hline \multicolumn{7}{|c|}{$\begin{array}{c}\text { TABLE-2 } \\
\text { RECOVERY OF THE THREE TROPANE ALKALOIDS }(n=9)\end{array}$} \\
\hline Component & Contents (mg) & Added (mg) & Determined (mg) & Recovery (\%) & Mean (\%) & RSD (\%) \\
\hline \multirow{9}{*}{ Anisodamine } & 0.0927 & 0.0403 & 0.040 & 98.7 & \multirow{9}{*}{97.3} & \multirow{9}{*}{1.4} \\
\hline & 0.0947 & 0.0403 & 0.040 & 100.2 & & \\
\hline & 0.0926 & 0.0403 & 0.039 & 97.2 & & \\
\hline & 0.0946 & 0.0806 & 0.0781 & 96.9 & & \\
\hline & 0.095 & 0.0806 & 0.0784 & 97.2 & & \\
\hline & 0.0946 & 0.0806 & 0.0773 & 95.9 & & \\
\hline & 0.0942 & 0.1210 & 0.116 & 95.9 & & \\
\hline & 0.0963 & 0.1210 & 0.117 & 96.7 & & \\
\hline & 0.0947 & 0.1210 & 0.118 & 97.5 & & \\
\hline \multirow{9}{*}{ Scopolamine } & 0.1254 & 0.0482 & 0.0490 & 100.7 & \multirow{9}{*}{103.0} & \multirow{9}{*}{1.5} \\
\hline & 0.1278 & 0.0482 & 0.0490 & 104.5 & & \\
\hline & 0.1225 & 0.0482 & 0.0500 & 100.6 & & \\
\hline & 0.1207 & 0.0965 & 0.101 & 104.5 & & \\
\hline & 0.1211 & 0.0965 & 0.101 & 104.9 & & \\
\hline & 0.1206 & 0.0965 & 0.0990 & 103 & & \\
\hline & 0.1201 & 0.145 & 0.147 & 101.6 & & \\
\hline & 0.1228 & 0.145 & 0.150 & 103.6 & & \\
\hline & 0.1207 & 0.145 & 0.150 & 103.4 & & \\
\hline \multirow{9}{*}{ Hyoscyamine } & 1.042 & 0.448 & 0.475 & 97.3 & \multirow{9}{*}{99.8} & \multirow{9}{*}{1.8} \\
\hline & 1.063 & 0.448 & 0.491 & 100.6 & & \\
\hline & 1.018 & 0.448 & 0.47 & 96.4 & & \\
\hline & 1.003 & 0.976 & 0.996 & 102.0 & & \\
\hline & 1.007 & 0.976 & 0.986 & 101.0 & & \\
\hline & 1.003 & 0.976 & 0.968 & 99.2 & & \\
\hline & 0.998 & 1.464 & 1.464 & 100.0 & & \\
\hline & 1.021 & 1.464 & 1.478 & 101.0 & & \\
\hline & 1.003 & 1.464 & 1.475 & 100.8 & & \\
\hline
\end{tabular}

TABLE-3

DETERMINATION OF TROPANE ALKALOIDS IN DIFFERENT FRACTIONS OF Herba belladonnae IN DIFFERENT SEASONS ( $\mathrm{n}=2$ )

\begin{tabular}{|c|c|c|c|c|c|}
\hline Sample No & Growth time (days) & Different parts & Hyoscyamine (mg/g) & Scopolamine (mg/g) & Anisodamine $(\mathrm{mg} / \mathrm{g})$ \\
\hline \multirow{4}{*}{1} & \multirow{4}{*}{120} & Root & 2.249 & 0.169 & 0.146 \\
\hline & & Stem & 1.561 & 0.592 & 0.0680 \\
\hline & & Leaf & 1.377 & 0.678 & 0.0780 \\
\hline & & Herb & 1.503 & 0.602 & 0.0790 \\
\hline \multirow{6}{*}{2} & \multirow{6}{*}{140} & Root & 1.484 & 0.251 & 0.125 \\
\hline & & Stem & 1.559 & 0.127 & 0.0130 \\
\hline & & Leaf & 3.068 & 0.962 & 0.128 \\
\hline & & Flowers & 3.061 & 1.396 & 0.159 \\
\hline & & Fruits & 3.728 & 1.587 & 0.504 \\
\hline & & Herb & 2.063 & 0.447 & 0.0781 \\
\hline \multirow{4}{*}{3} & \multirow{4}{*}{150} & Root & 0.383 & 0.190 & 0.0752 \\
\hline & & Stem & 1.005 & 0.217 & 0.0772 \\
\hline & & Leaf & 1.855 & 0.763 & 0.135 \\
\hline & & Herb & 1.219 & 0.413 & 0.0961 \\
\hline \multirow{6}{*}{4} & \multirow{6}{*}{160} & Root & 2.867 & 0.236 & 0.182 \\
\hline & & Stem & 1.811 & 0.197 & 0.129 \\
\hline & & Leaf & 3.852 & 0.743 & 0.206 \\
\hline & & Flowers & 3.305 & 0.585 & 0.162 \\
\hline & & Fruits & 2.501 & 0.446 & 0.0740 \\
\hline & & Herb & 2.534 & 0.348 & 0.1540 \\
\hline \multirow{6}{*}{5} & \multirow{6}{*}{170} & Root & 1.788 & 0.178 & 0.0980 \\
\hline & & Stem & 1.129 & 0.149 & 0.0710 \\
\hline & & Leaf & 3.249 & 0.834 & 0.215 \\
\hline & & Flowers & 4.058 & 0.733 & 0.211 \\
\hline & & Fruits & 2.821 & 0.707 & 0.197 \\
\hline & & Herb & 1.872 & 0.349 & 0.118 \\
\hline \multirow{6}{*}{6} & \multirow{6}{*}{180} & Root & 2.554 & 0.200 & 0.298 \\
\hline & & Stem & 1.222 & 0.128 & 0.095 \\
\hline & & Leaf & 4.168 & 0.939 & 0.375 \\
\hline & & Flowers & 5.111 & 0.727 & 0.344 \\
\hline & & Fruits & 2.539 & 0.561 & 0.205 \\
\hline & & Herb & 2.268 & 0.362 & 0.204 \\
\hline
\end{tabular}




\section{Conclusion}

In this study, three main tropane alkaloids were investigated in different fractions of Herba belladonnae collected in various seasons. To the best of our knowledge, it is the first report which simultaneously determined the three main tropane alkaloids in different fractions of Herba belladonnae collected in various seasons, not only quantitatively but also qualitatively. Quantitative analysis showed that the contents of three main tropane alkaloids mainly distributed in leafs, flowers and fruits, the contents of hyoscyamine in leaves and flowers were the highest, followed by fruits and roots and the third was stem; the contents of scopolamine in flowers and fruits were the highest; the contents of anisodamine in fruits were the highest and the contents of the 3 components appeared an upgrade trend during the growing period and reached to a balance in the harvest time. The study on dynamic changes of the three main tropane alkaloids in different parts of Herba belladonna in different growing periods could provide a guidance for the plantation, harvest and scientific evaluation of Herba belladonna.

\section{REFERENCES}

1. Chinese Pharmacopoeia, Vol. I, p. 355 (2010).

2. K.S. Wang, Plants, 26, 26 (2003).

3. K. Sun and L.M. Yang, World Clin. Drugs, 31, 182 (2010).

4. S.Q. Liu, W.J. Zang, Z.L. Li, Q. Sun and X.J. Yu, J. Xi'an Jiaotong Univ. (Med. Sci.), 26, 16 (2005).

5. A. Lange and P. Toft, Ugeskr Laeger, 152, 1096 (1990).

6. U. Cikla, S. Turkmen, Y. Karaca, F.A. Ayaz, S. Turedi and A. Gunduz, Hum. Exp. Toxicol., 30, 1998 (2011).

7. F. Laffargue, C. Oudot, A. Constanty, A. Bedu and S. Ketterer-Martinon, Arch. Pediatr., 18, 186 (2011).

8. E.P. Krenzelok, Clin. Toxicol., 48, 104 (2010).

9. J. Kupper and C. Reichert, Ther. Umsch, 66, 343 (2009).

10. A.M. Montoya, N. Mavrakanas and J.S. Schutz, Eur. J. Ophthalmol., 19, 170 (2009).

11. Chinese Pharmacopoeia, Vol. II, p. 560 (2010). 